\title{
Development of CT Texture Analysis in COPD and Association with Visual Scoring and $\mathrm{DL}_{c o}$
}

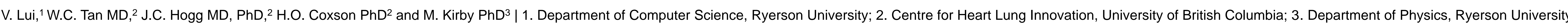

\section{Rationale}

- In chronic obstructive pulmonary disease (COPD) patients, emphysema severity can be assessed using computed tomography (CT) qualitatively by Radiologist scoring and quantitalively using hislogram based densily hrosholding

- However, studies have shown that qualitative CT emphysema scores predict outcomes such as lung cancer risk while quantitative $\mathrm{CT}$ emphysema does not

Qualitative CT emphysema scoring may therefore capture other information that is related to emphysema severity besides amount of emphysema, such as lesion size or clustering of emphysena, spalfal distibution and helerogenely

- Novel textural constructs, such as gray level co-occurrence matrix (GLCM) and fracta dimensions (FD) have been used in various applications in medical imaging and migh

\section{Objective \& Hypothesis}

objectives

To determine if $\mathrm{CT}$ texture features, such as GLCM and FD, can differentiate patients with COPD from healthy volunteers, and are related to lung function

- To determine if $C T$ texture features are association with qualitative visual scoring

- To determine if CT texture features are significantly associated with COPD outcomes, independent of qualitative scoring and standard quantitative $\mathrm{CT}$ emphysema measurements Hypothesis

- CT texture features can be developed to objectively aid in quantifying the severity of emphysema, and may provide information complementary to qualitative visual assessment

\section{Methods}

Study Population

- Clinical data and CT imaging from the visit 1 Canadian Cohort Obstructive Lung Disease (CanCOLD) study were utilized

- A total of 1187 subjects were used based on the completeness of their clinical and imaging

- COPD severity was defined using the Global initiative for Chronic Obstructive Lung Disease

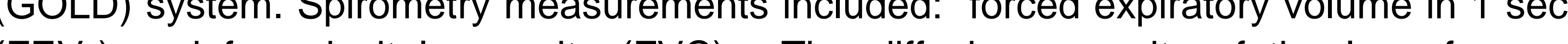
and forced Vilifusion capacity of the lung for carbon

- CT images were visually scored by a trained radiologist on a scale of $0-4$, emphysema prevalence is a binary variable of $0 / 1$ indicating none/visible

Table 1. Subject Demographics

\begin{tabular}{|c|c|c|c|c|}
\hline Parameter & $\begin{array}{c}\text { Never-smoker }(\mathrm{N}) \\
(\mathrm{n}=258)\end{array}$ & $\begin{array}{l}\text { At-Risk (R) } \\
(\mathrm{n}=255)\end{array}$ & $\begin{array}{c}\text { GOLD IG1) } \\
(\mathrm{n}=4111)\end{array}$ & $\begin{array}{l}\text { GOLD DIt (G2) } \\
(\mathrm{n}=263)\end{array}$ \\
\hline $\begin{array}{lll}\text { Sex }(\%) & \text { Male } & \text { Female }\end{array}$ & & & $62 \%$ & $\begin{array}{l}54 \% \\
6 \% \\
\end{array}$ \\
\hline 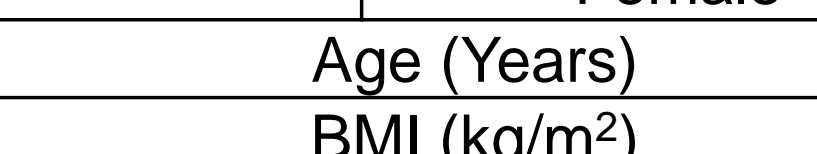 & 66.90 & 66.57 & 67.06 & 66.14 \\
\hline 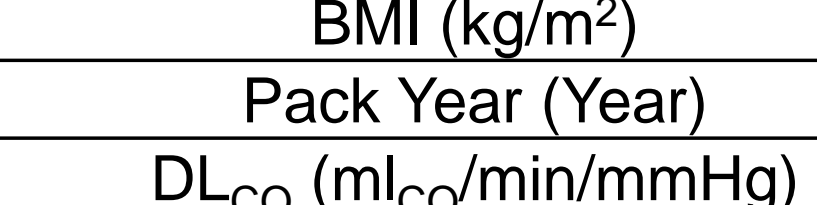 & 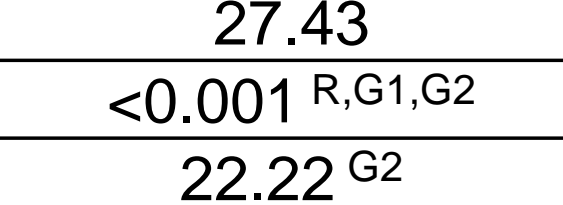 & 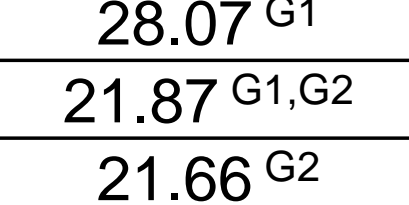 & $\begin{array}{l}\frac{26.9062}{17.562} \\
21.9 \sigma^{62}\end{array}$ & $\begin{array}{l}2 \frac{2.32}{27.13} \\
19.46\end{array}$ \\
\hline
\end{tabular}

Imaging Processing and Feature Selection

Standard Quantitative CT (QCT) Measurements:

The low attenuation areas of the lung below $-950 \mathrm{HU}\left(\mathrm{LAA}_{950}\right)$ and the and the low attenuation cluster (LAC) were generated

Texture Measurements:

GLCM'12,13 consisting of Hounsfield unit from -1 to -1000 HU were created from the CT lung image, for which 23 texture features were extracted using MATLAB $22019 \mathrm{ga}$. Those includes and Clausi's ${ }^{16} .10 \mathrm{FD}$ first order statistics were derived through the image processing of the $\mathrm{CT}$ lung images via the boxcount ${ }^{17}$ method in examining $16 \times 16 \times 16$ window increments with Blockproc3D ${ }^{18}$.

\section{Statistical Analysis:}

Feature selection was performed using the generalized orthogonal matching pursuit (gOMP) algorithm via the MXM package using $R$

- Statistical significance was determined via multivariate regressions models with selected features as the predictors and $\mathrm{DL}_{\mathrm{co}}$ as response

\section{Results}

Table 2. Features examined from $\mathrm{CT}$ lung images

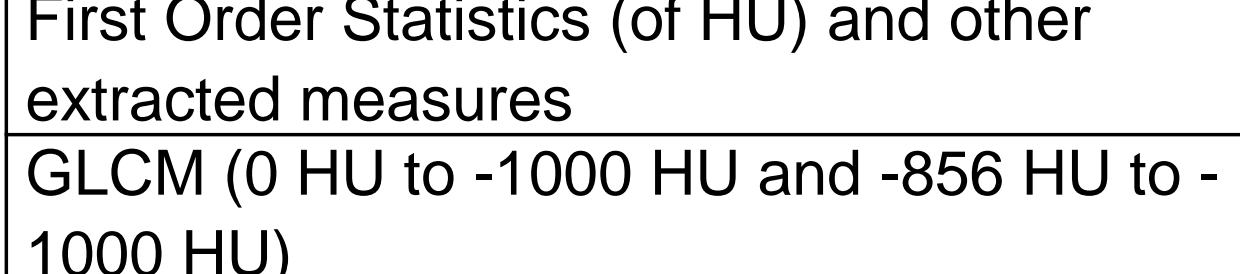

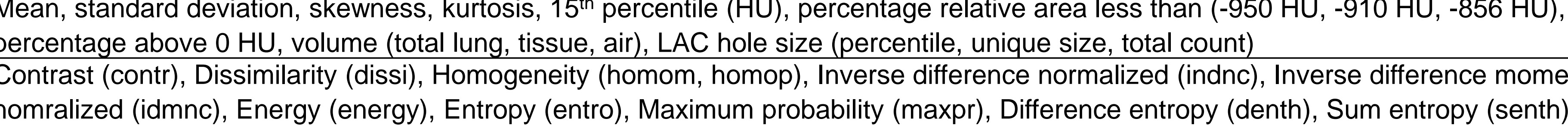

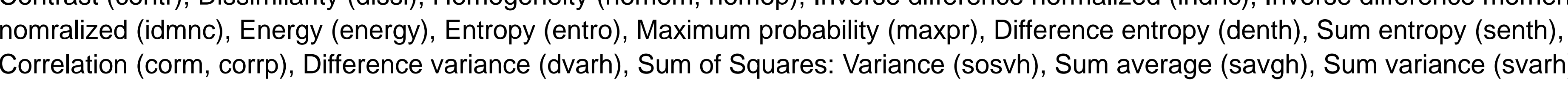

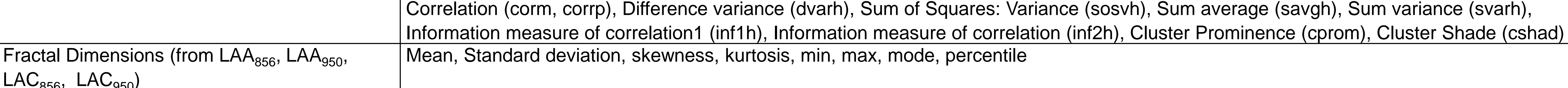

Imaging Biomarker Development: Are CT texture measurements associated with COPD severity?

Table 3 Pearson's correlation plot of $\mathrm{CT}$ texture features with lung function and ANOVA

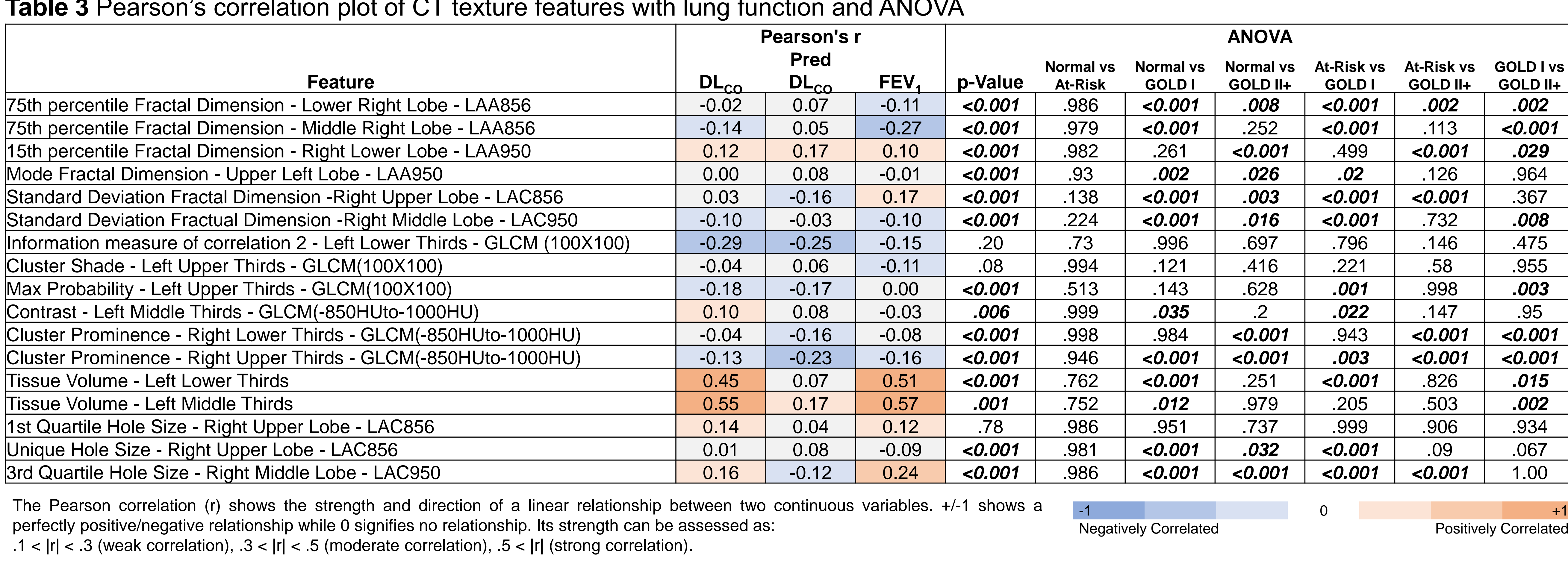

Are CT texture measurements associated with radiologist's visual score of emphysema prevalence?

Table 4.1. shows that in the covariates + QCT +

CT texture model, QCT provided no additional

information for predicting visual emphysema.

Models with CT texture were shown to have greater AUC and lower AIC. Additionally, Table
4.2 shows increase in the cluster prominence of 4.2 shows increase in the cluster prominence of showed the greatest increases in the odds ratio

Table 4.1. Logistic multivariable regression model for $\mathrm{QCT}$ and $\mathrm{CT}$ texture predictors with emphysema prevalence as response: Model: Emphysema Prevalence Score $+C T$ texture measurements
+ QCT $+C T$ texture measurements

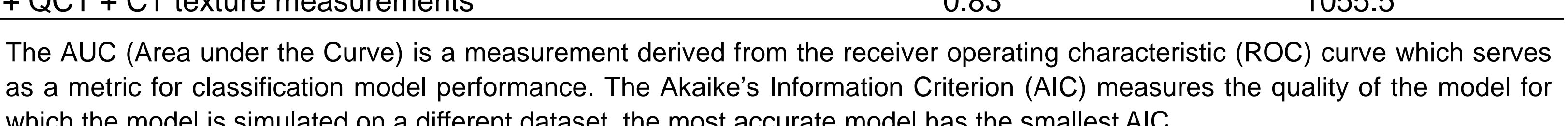
emphysema prevalence.

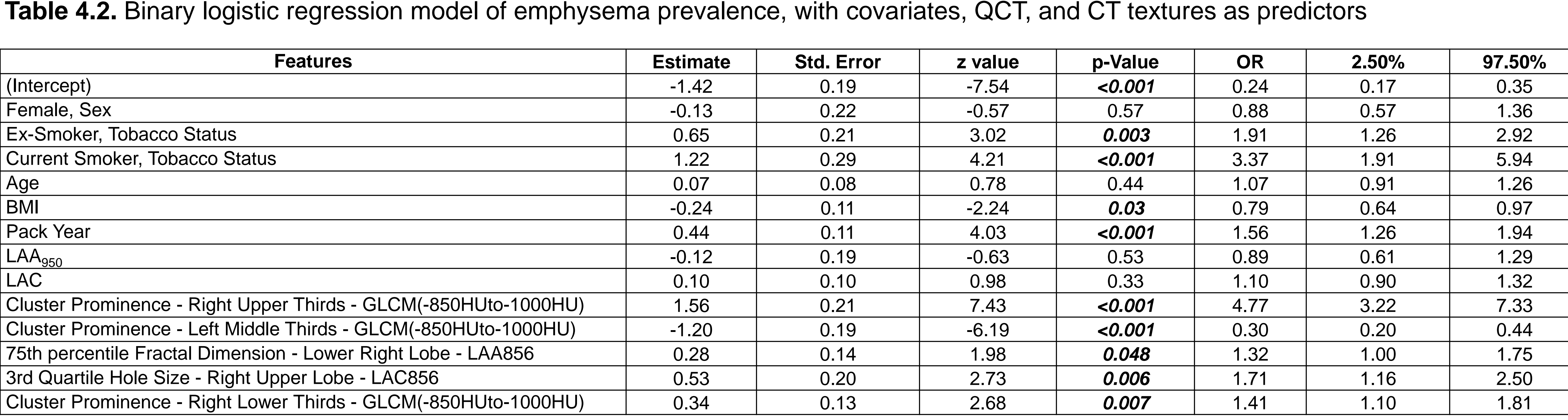

Are $C T$ texture measurements associated with $D L_{c o}$, independent of radiologist's visual score of emphysema presence? Both visual score and QCT provided Table 5.2. Linear regression model of $\mathrm{DL}_{\mathrm{co}}$ with visual score, $L A A_{950}, \mathrm{CT}$ texture independent and significant association to $\mathrm{DL}_{\mathrm{co}}$. When $\mathrm{CT}$ textures were added Into the model, QCT no longer remained score provided textures and visual significant association with $\mathrm{DL}_{\mathrm{co}}$

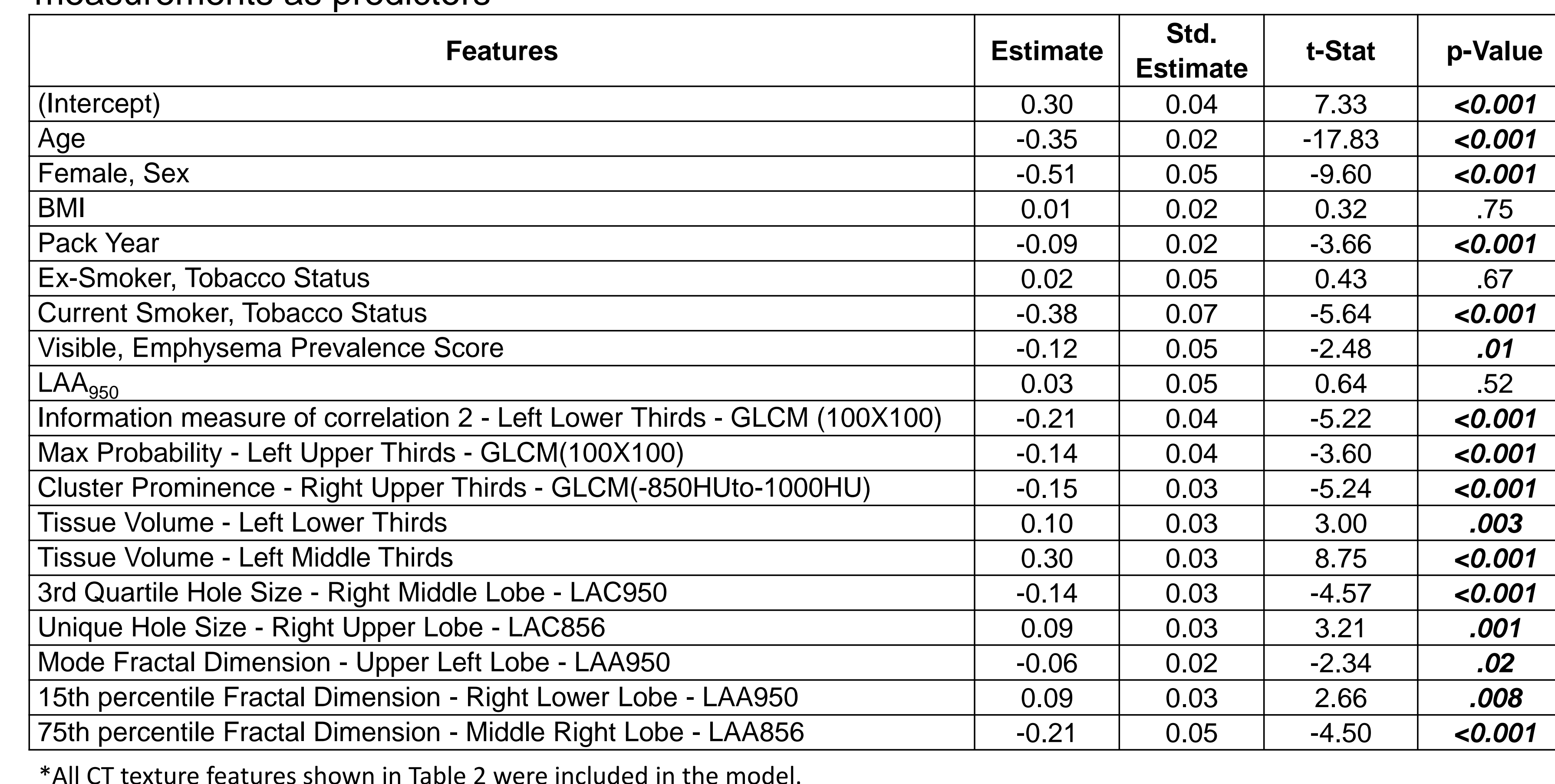

Discussion \& Conclusions

- CT texture measurements significantly
differentiated subjects with COPD, and were significantly associated with lung function measurements

CT texture measures were significantly associated with qualitative visual emphysema prevalence, and wereastronger predictors than standard quantitative LA

- In a multivariable regression model for $\mathrm{DL}_{\mathrm{CO}_{0}}, \mathrm{CT}$ texture measurements and qualitative visual score were significant prediclors, but standard quantitative CT were no longer significant in

Both visual emphysema scoring and $C T$ texture complementary information related to pulmonary

\section{Future Directions}

Further investigation of the use of $\mathrm{CT}$ texture features is required, such as:

Can CT texture features predict longitudinal emphysema progression?

Are $\mathrm{CT}$ texture features associated with different emphysema subtypes, such as panlob
paraseptal and centrilobular emphysema? Are $\mathrm{CT}$ texture features and emphysema subtype
classification independently associated with
pulmonary function decline in COPD?

\section{References}

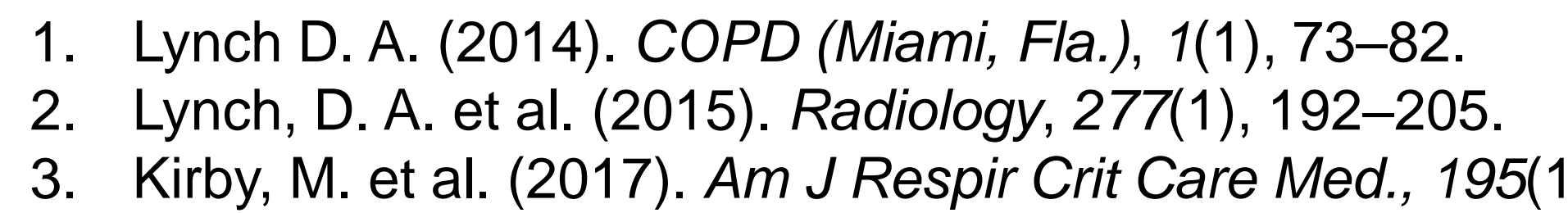
Kirby, M. et al. (2017). Am JRespir Crit Care Med, 195(1).
4. Kim, S. S, et al. (2013). Radiology, 266(2).
Mascalchi, M. Camiciottoli, G., \& Diciotiti, S. (2017). J J Thorac

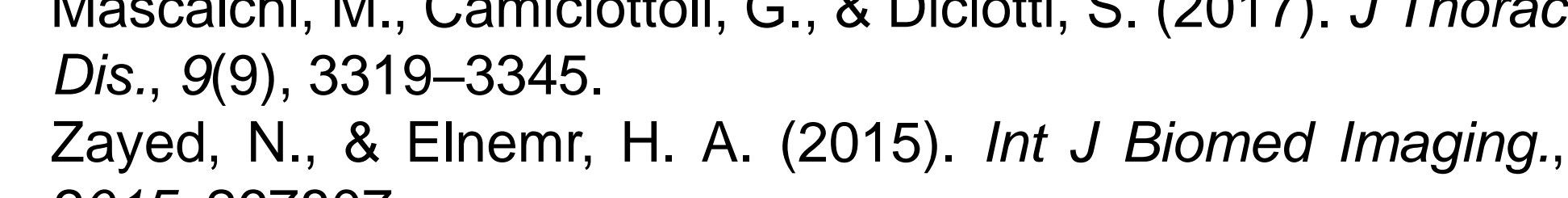
2015, 267807.

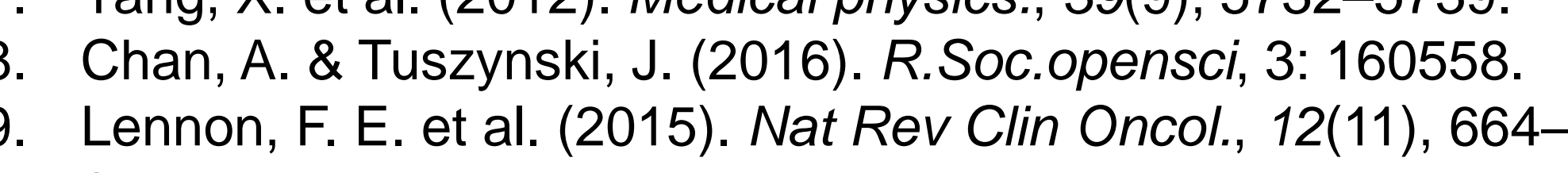
0. Bourbeau, J. et al. (2014). COPD, 112, 125-132.

Lewandowski, Z. \& Beyenal, H. (2007). MATLAB Central File 3. Uopplutiri. A.(2008). MATLAB Central File Exchange.
14. Haralic, R.M., Shanmugam, K. \& Dinstein, I. (1973). IEEE

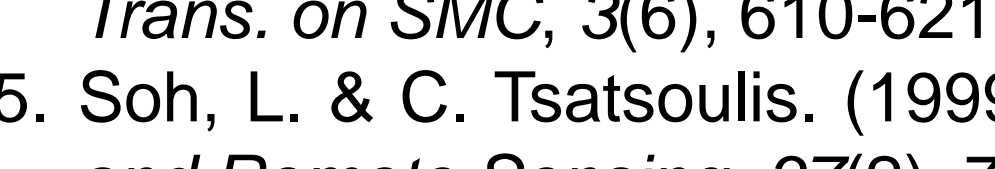
and Remote Sensing, 37(2), 780-795 Trans. on Geoscience 6. DA. Clausi,, (2002). Can. J. Remote Sensing, 28(1), 45-62. 18. Cassero, R. (2011). Gitthub

\section{Acknowledgments}

would like to thank the Faculty of Science (URO) for salary support. I would like to personally hank you for the support and guidance from Dr. M. Kinby, Jason, Daniel, Danesh, and Diana in this acknowledge all the researchers, staff and subjects in the CanCOLD study as well as VIDA Diagnostic.

\begin{tabular}{|c|c|}
\hline 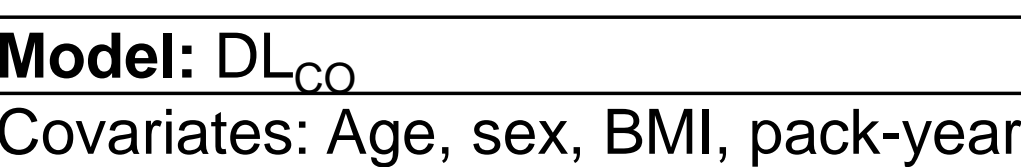 & $\begin{array}{l}\text { Adjusted } R^{2} \\
017\end{array}$ \\
\hline 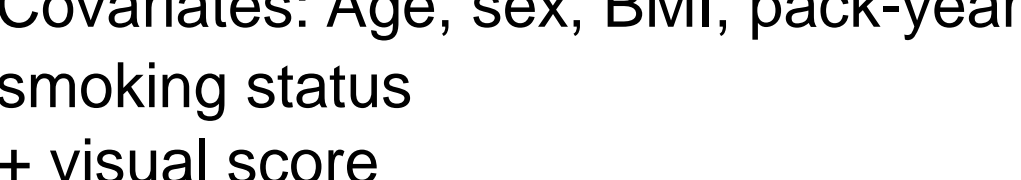 & 0.47 \\
\hline 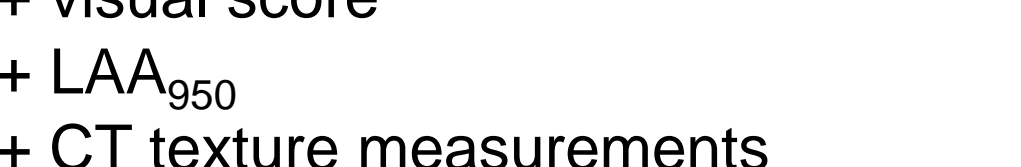 & 0.47 \\
\hline 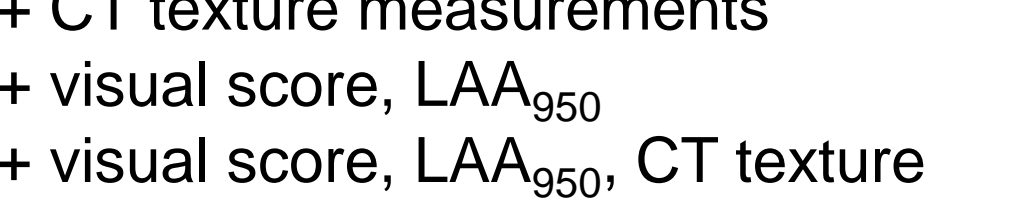 & 0.48 \\
\hline measurements & \\
\hline
\end{tabular}

Faculty of Science 